\title{
Study on Improving the Turbidity Measurement of the Absolute Coagulation Rate Constant
}

\author{
Zhiwei Sun,* Jie Liu, and Shenghua Xu \\ NML, Institute of Mechanics, Chinese Academy of Sciences, Beijing 100080, PR China
}

Received January 23, 2006. In Final Form: March 27, 2006

\begin{abstract}
The existing theories dealing with the evaluation of the absolute coagulation rate constant by turbidity measurement were experimentally tested for different particle-sized (radius $=a$ ) suspensions at incident wavelengths $(\lambda)$ ranging from near-infrared to ultraviolet light. When the size parameter $\alpha=2 \pi a / \lambda>3$, the rate constant data from previous theories for fixed-sized particles show significant inconsistencies at different light wavelengths. We attribute this problem to the imperfection of these theories in describing the light scattering from doublets through their evaluation of the extinction cross section. The evaluations of the rate constants by all previous theories become untenable as the size parameter increases and therefore hampers the applicable range of the turbidity measurement. By using the T-matrix method, we present a robust solution for evaluating the extinction cross section of doublets formed in the aggregation. Our experiments show that this new approach is effective in extending the applicability range of the turbidity methodology and increasing measurement accuracy.
\end{abstract}

\section{Introduction}

Knowledge of the stability and coagulation kinetics of colloidal systems is crucial for various applications in chemical, food, pharmaceutical, and material industries, environmental engineering, biotechnology, and nanotechnology. ${ }^{1-7}$ The absolute coagulation rate constant is an important parameter used to characterize the coagulation kinetics of colloidal systems, and accurate experimental data are particularly important for the development of relevant theories or for deriving information about colloidal interactions. To determine the absolute coagulation rate constant of suspensions, the turbidity measurement has been extensively adopted 8,9 because of its simplicity and easy implementation.

However, to determine the absolute coagulation rate by the turbidity measurement, the difference between extinction (or attenuation) cross sections of one doublet or dimer (two adhered particles) and of two single particles has to be evaluated from theory. For spheres, the extinction cross section can be calculated exactly from Mie theory. However, for hard sphere particles (e.g., polystyrene latex) considered in our study, the doublets are not spherical, so it cannot be calculated exactly by Mie theory. Also, the existing theories used to calculate the extinction cross sections of doublets for treating the turbidity measurement are valid only for small particles. Thus, until now the application of this methodology has been limited to suspensions of small particles. ${ }^{9}$

* To whom correspondence should be addressed. E-mail: sunzw@ imech.ac.cn.

(1) Allain, C.; Cloitre, M.; Wafra M. Phys. Rev. Lett. 1995, 74, 1478.

(2) Molina-Bolívar, J. A.; Galisteo-González, F.; Hidalgo-Álvarez, R. J. Chem. Phys. 1999, 110, 5412.

(3) R. Finsy, E.; Moreels, Bottger, A.; Lekkerkerker, H. J. Chem. Phys. 1985, 82,3812 .

(4) Stanislav, D.; Chao, Z.; Rajesh N. D.; Qun, Y. Adv. Colloid Interface Sci. 2005, 114, 119.

(5) Vincent, B. Adv. Colloid Interface Sci. 1992, 42, 279.

(6) Sun, Z. W.; Xu, S. H.; Dai, G. L.; Li, Y. M.; Lou, L. R.; Liu Q. S.; Zhu, R. Z. J. Chem. Phys. 2003, 119, 2399.

(7) Sun, Z. W.; Xu, S. H.; Liu, J.; Li, Y. M.; Lou L. R.; Xie, J. C. J. Chem. Phys. 2005, 122, 184904.

(8) Elimelech, M.; Gregory, J.; Jia, X.; Williams, R. A. Particle Deposition and Aggregation; Butterworth-Heinemann: Oxford, England, 1995.

(9) Lichtenbelt, J. W. Th.; Ras, H. J. M. C.; Wiersema, P. H. J. Colloid Interface Sci. 1974, 46, 522.
To improve the applicability of the turbidity measurement, in this study the T-matrix method ${ }^{10-13}$ is proposed to accurately calculate extinction cross sections of real doublets in the turbidity measurement. In addition, we describe a special experimental approach using different particle-sized (radius $=a$ ) suspensions at incident wavelengths $(\lambda)$ ranging from near-infrared to ultraviolet light to expose the physical nature reflected in the turbidity measurement and to examine the performances of different theoretical approaches. Our experiments show that the rate constants derived from all previous theories become untenable as the size parameter $(\alpha=2 \pi a / \lambda)$ increases. In contrast to these existing theories, the T-matrix method provides a robust solution that is effective in extending the applicability range of the turbidity methodology and increasing measurement accuracy.

\section{Theory}

In the earliest stage of the coagulation process for a monodisperse colloidal system, only collisions of single particles to form doubles need to be considered. Therefore, the change in particle number concentration can be approximately expressed as

$$
\begin{gathered}
\left(\frac{\mathrm{d} N_{1}}{\mathrm{~d} t}\right)_{t=0}=-k_{11} N_{1}^{2} \\
\left(\frac{\mathrm{d} N_{2}}{\mathrm{~d} t}\right)_{t=0}=\frac{k_{11} N_{1}^{2}}{2}
\end{gathered}
$$

where $N_{1}$ and $N_{2}$ are the number concentrations of single particles and doublets, respectively, $t$ is time, and $k_{11}$ is the coagulation rate constant.

Because the turbidity is expressed as $\tau=N_{1} C_{1}+N_{2} C_{2}$, where $C_{1}$ and $C_{2}$ are the extinction cross sections for the single particle and doublet, respectively, and $\tau$ is the turbidity, the change rate

(10) Mishchenko, M. I.; Travis, L. D.; Lacis, A. A. Scattering, Absorption, and Emission of Light by Small Particles; Cambridge University Press: Cambridge, U.K., 2002.

(11) Quirantes, A.; Delgado, A. J. Quant. Spectrosc. Radiat. Transfer 2003, 78,179 .

(12) Mishchenko, M. I. J. Opt. Soc. Am. A 1991, 8, 871.

(13) Mackowski D. W. J. Opt. Soc. Am. A 1994, 11, 2851. 
in turbidity due to the aggregation of single particles can be written as

$$
\frac{\mathrm{d} \tau}{\mathrm{d} t}=C_{1} \frac{\mathrm{d} N_{1}}{\mathrm{~d} t}+C_{2} \frac{\mathrm{d} N_{2}}{\mathrm{~d} t}
$$

Combining eqs 1,2 , and 3 , the coagulation rate constant can be connected to the rate of turbidity change in the turbidity measurement by the following equation ${ }^{9}$

$$
k_{11}=\frac{\left[\mathrm{d}\left(\tau / \tau_{0}\right) / \mathrm{d} t\right]_{0}}{\left[\left(C_{2} / 2 C_{1}\right)-1\right] N_{1}}
$$

where $\tau_{0}$ is the turbidity at the coagulation starting time $t=0$. Assuming the denominator in eq 4 can be taken to be a constant, the coagulation rate will be proportional to the quantity $R=$ $\left[\mathrm{d}\left(\tau / \tau_{0}\right) / \mathrm{d} t\right]_{0}$, the relative rate of turbidity change. Thus, $|R|$ is usually used to represent the relative coagulation rate. The dimensionless parameter $F=\left[\left(C_{2} / 2 C_{1}\right)-1\right]$ is referred to the optical factor. $R$ can be obtained from the turbidity measurement, but the optical factor has to be calculated by means of light scattering theory.

The calculation of the optical factor is involved in the evaluation of extinction cross sections for single particles and doublets. Because the light scattering from a sphere can be well solved by Mie theory, the key problem is how to describe the light scattering correctly from doublets of two hard sphere particles through their evaluation of the extinction cross section.

Lichtenbelt et al. ${ }^{9}$ compared different theoretical approaches to acquire the optical factor and made some modification to the theories to improve their performance. These theories include RGD theory, Mie theory with the coalescing assumption of supposing two coagulated particles form a spherical doublet with the same volume, RGD with the coalescing assumption, and corrected RGD theory. ${ }^{9}$ In short, we will refer to them below as RGD(real), Mie(coalescence), RGD(coalescence), and RGD(corrected), respectively. RGD theories are based on the theory of Rayleigh scattering and are therefore applicable only to small particles. Mie scattering has no size limitations but is basically restricted to the case of a perfect sphere. Therefore, all of the theories dealing with the turbidity measurement mentioned above either cannot treat large particles correctly or have to resort to the coalescing assumption. ${ }^{9}$ Thus, the measurement of the coagulation rate constant by using the turbidity measurement is suitable only for small particles.

To evade the difficulty in directly evaluating the light scattering intensity from a doublet, a technique of simultaneous static and dynamic light scattering ${ }^{14-16}$ has been developed to measure the absolute rate constants. This technique allows the factors related to the scattering intensity from a doublet appearing in the analogous expressions for static and dynamic light scattering to be eliminated. Apparently, this technique is much more complicated than the turbidity measurement. This study will explore only the possibility of improvement in the determination of the coagulation rate constant by using the turbidity measurement.

As discussed above, the key problem in improving the turbidity measurement of the rate constant is how to evaluate the extinction cross section of a real doublet accurately. Concerning this issue, the T-matrix technique presents a very promising solution because

(14) Holthoff, H.; Egelhaaf, S. U.; Borkovec, M.; Schurtenberger, P.; Sticher, H. Langmuir 1996, 12, 5541 .

(15) Lin, W.; Galletto, P.; Borkovec, M. Langmuir 2004, 20, 7465.

(16) Berka, M.; Rice, J. A. Langmuir 2004, 20, 6152. of its great capabilities in accurately computing electromagnetic scattering by single and compounded particles without a size limit.

In the T-matrix method, both incident and scattered electric fields are expanded in a series of vector spherical wave functions, and the scattered field coefficients are related to the incident field coefficients by means of the so-called transition matrix (or T matrix). From the transition matrix, the extinction cross sections of single particles and doublets, and thus the optical factor $F$, can be calculated without additional assumptions that Mie theory and RGD theory use. An outline of using the T matrix to evaluate the extinction cross section is described in the Appendix. A more comprehensive discussion of the technical details and calculated data of the optical factor for a range of particle radii and incident light wavelengths will be presented in a separate publication.

\section{Experimental Section}

Three types of polystyrene latex particles with a density $\rho=1.05$ $\mathrm{g} / \mathrm{cm}^{3}$ were used in this study. The particle radii used in the experiments are $a=170,250$, and $500 \mathrm{~nm}(3000-4000$ series product from Duke Scientific Corporation). The initial particle number concentration of the stock solution was determined according to the particle size and dry weight of the dispersions of a certain amount of the sampling solution. The estimated error associated with the number concentration is about $10 \%$.

A UV-vis dual-beam spectrophotometer (Purkinje TU-1901, Beijing) was employed to measure the coagulation rates of all of the latexes. A computer was connected to the instrument to collect data. All results presented below are the values averaged over five independent experiments.

Before the measurements were started, the containers for the electrolyte and latex solutions as well as the sample cells were cleaned with a chromium sulfuric acid solution in order to eliminate organic materials. The use of any detergent-based cell-cleaning solution was avoided because the coagulation rate constant is sensitive to the presence of trace amounts of surfactants. Water was obtained from an ion-exchange apparatus, and the conductivity is lower than 0.5 $\mu \mathrm{S} / \mathrm{cm}$.

The experiments were performed at a temperature of $T=25^{\circ} \mathrm{C}$, and for each sample, the fast coagulation was initiated by mixing $1 \mathrm{~mL}$ of electrolyte solution (having a concentration sufficiently higher than the required critical coagulation concentration) with 1 $\mathrm{mL}$ of latex solution in the sample cell (path length $=10 \mathrm{~mm}$ ) for all of the experiments. The final number concentrations of particles in the cell are $1 \times 10^{14}$ and $5 \times 10^{14} \mathrm{~m}^{-3}$ for particles of $a=500$ and $250 \mathrm{~nm}$, respectively, and $5 \times 10^{15} \mathrm{~m}^{-3}$ for particles of $a=170$ nm.

For all of the experiments, the data of transmission percentage $(T \%)$ versus time was recorded ${ }^{6}$ during the coagulation process for different particle-sized polystyrene (PS) dispersions with an initial number concentration $\left(N_{1}\right)$ at incident wavelengths ranging from near-infrared to ultraviolet light. (The wavelength in vacuum was from 250 to $870 \mathrm{~nm}$ in our experiments.) Then, the transmission percentages were transformed to turbidity by the equation

$$
\tau=-\left(\frac{1}{L}\right)(\ln T \%)
$$

where $L$ is the path length through the dispersion. The slope of the $\tau / \tau_{0}$ versus time plot (the relative coagulation rate) was calculated by the procedure of linear regression in the early stages of coagulation to get the absolute coagulation rate constant $\left(k_{11}\right)$ from eq 4.

To ensure that the experiments were really done in the early stages, the experimental window should be much smaller than the coagulation time or half-life $t_{1 / 2}$, which is the time required for the reduction of the number of particles by one-half. The theoretical 


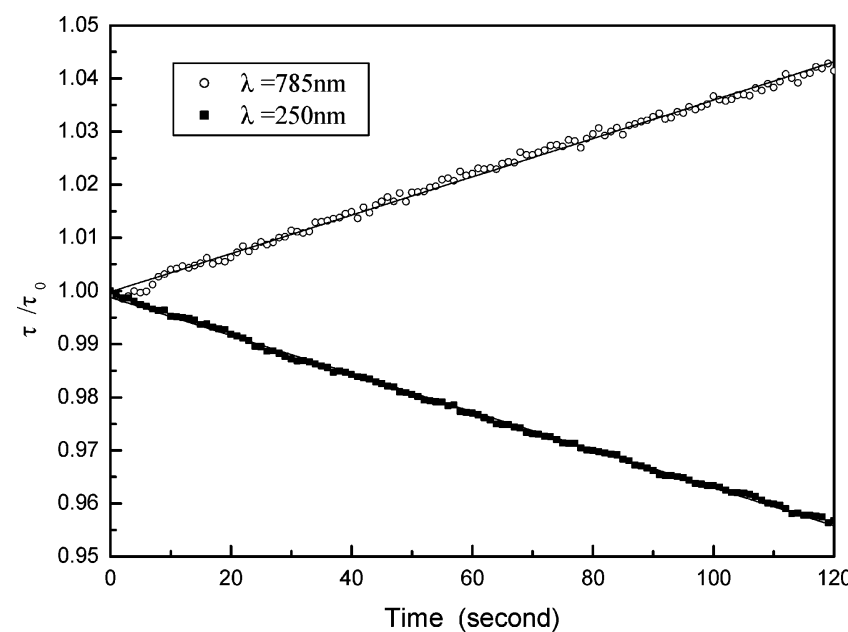

Figure 1. Plot of relative turbidity $\tau / \tau_{0}$ vs time $t$ for suspensions of particles with radius $a=250 \mathrm{~nm}$ at incident wavelengths (particle number concentration $N_{1}=5 \times 10^{14} \mathrm{~m}^{-3}$ ).

coagulation time is given by ${ }^{17}$

$$
t_{1 / 2}=\frac{3 \eta}{4 k T N_{1}}
$$

where $\eta$ and $k$ are the viscosity and Boltzmann constant, respectively. More specifically, for the case of water as the medium at $T=25$ ${ }^{\circ} \mathrm{C}$, we simply have ${ }^{18}$

$$
t_{1 / 2} \approx \frac{2 \times 10^{17}}{N_{1}}(\text { seconds })
$$

Using eq 7, the coagulation times for particles $a=500,250$, and $170 \mathrm{~nm}$ are 2000,400 , and $40 \mathrm{~s}$, respectively. Therefore, the measurement durations in our experiments are 300,60 , and $8 \mathrm{~s}$ for the three kinds of particles, respectively, which are much shorter than the coagulation time. Taking into account the fact that the actual coagulation time was always longer than the theoretical value, we assume that the experiments were done in the early stages of coagulation.

\section{Results and Discussion}

Figure 1 gives two typical $\tau / \tau_{0}$ versus $t$ lines at two different wavelengths for suspensions consisting of PS particles with $a$ $=250 \mathrm{~nm}$ (particle number concentration $N_{1}=5 \times 10^{14} \mathrm{~m}^{-3}$ ). According to this type of plot, $R$ can be evaluated by linear fitting techniques for its linear portion near $t \approx 0$. We can see that even for the same dispersions the slope of the line of $\tau$ versus $t$ may be positive or negative depending on the wavelength $\lambda$ used.

Figure 2 shows a plot of the relative turbidity $\tau / \tau_{0}$ versus time $t\left(\tau / \tau_{0} \approx t\right)$ for suspensions of particles with radius $a=170 \mathrm{~nm}$ at an incident wavelength of $700 \mathrm{~nm}\left(N_{1}=5 \times 10^{15} \mathrm{~m}^{-3}\right)$. Compared with particles of $a=250 \mathrm{~nm}$ shown in Figure 1, the line of $\left(\tau / \tau_{0} \approx t\right)$ becomes obviously curved, as indicated by its shorter coagulation time, because the number concentration has to be increased for smaller particles as a result of detection problems (as will be discussed below). Apparently, the short linear portion of the $\left(\tau / \tau_{0} \approx t\right)$ curve will cause larger experimental errors.

The plot in Figure 3 shows the dependence of $R$ on $\lambda$ (as well as $\alpha$ ) for suspensions consisting of PS particles $(a=250 \mathrm{~nm})$.

(17) Smoluchowski, M. v. Phys. Z. 1916, 17, 557. Smoluchowski, M. v. Phys. Z. 1916, 17, 585. Smoluchowski, M. v. Z. Phys. Chem. 1917, 92, 129.

(18) Myers, D. Surfaces, Interfaces, and Colloids: Principles and Applications, 2nd ed.; John Wiley \& Sons: New York, 1999.

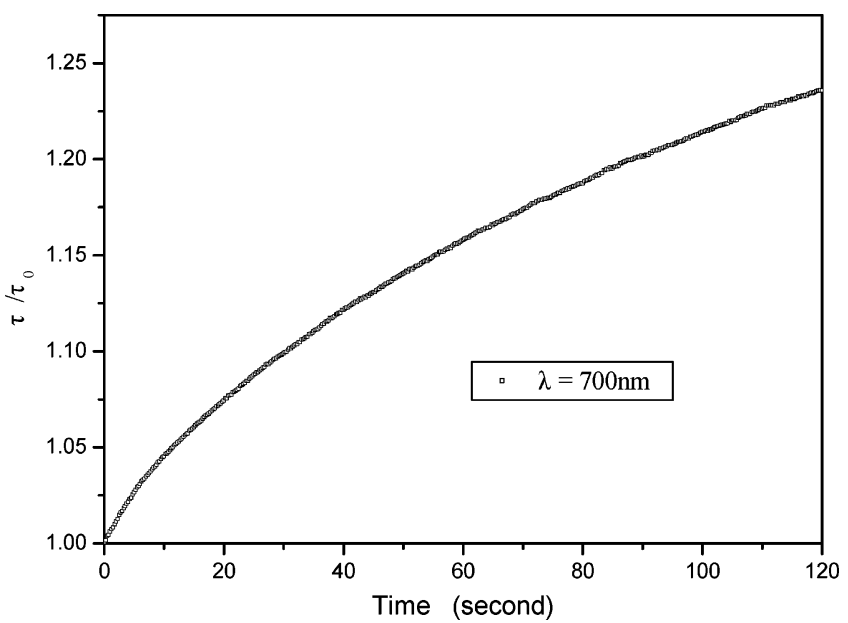

Figure 2. Plot of relative turbidity $\tau / \tau_{0}$ vs time $t$ for suspensions of particles with radius $a=170 \mathrm{~nm}$ at incident wavelengths (particle number concentration $N_{1}=5 \times 10^{15} \mathrm{~m}^{-3}$ ).

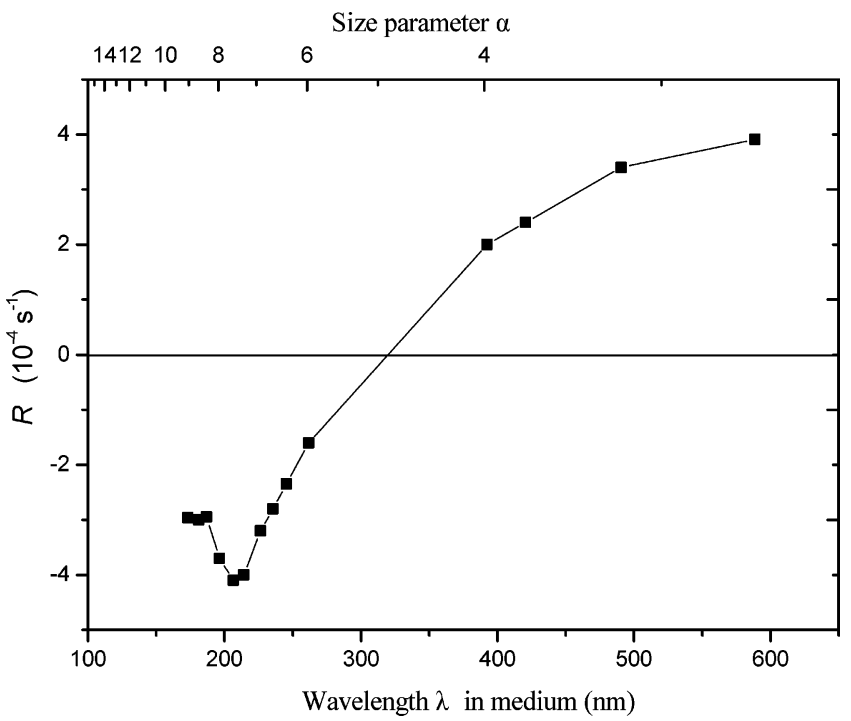

Figure 3. Relative rate of turbidity change $R=\left(1 / \tau_{0}\right)(\mathrm{d} \tau / \mathrm{d} t)_{0}$ at $t \rightarrow 0$ vs wavelength $\lambda$ and size parameter $\alpha$.

From Figure 3, we can see that $R$ varies dramatically with $\lambda$ : not only is its magnitude very different at different $\lambda$ but its signs also may change from negative through zero to positive. When two primary particles are combined to form a doublet during coagulation, there will be a corresponding change in their extinction sections. If the extinction section of two singlets $\left(2 C_{1}\right)$ is less than that of one doublet $\left(C_{2}\right)$, then we will have $(\mathrm{d} \tau / \mathrm{d} t)$ $>0$, namely, $R>0$; otherwise, $R<0$. And the case of $R=0$ corresponding to the extinction section of two singlets is equal to that of one doublet. If the measurement is performed at $\lambda$ with $R \approx 0$, then there will be no $R$ response to the actual coagulation process. This means that in the zone of $\lambda$ with $R \approx 0$ the change in the turbidity during coagulation is completely insensitive to the change in the particle number $N$ of the suspensions. That is the reason that there are no data points marked in the plot of Figure 3 around $\lambda=320 \mathrm{~nm}$ (the zero-sensitivity zone or the blind zone), where $R$, the magnitude of the slope of the $\tau-\mathrm{t}$ curve at $t=0$, is too small to distinguish (the signal is covered by noise). Thus, to enhance the signal-to-noise ratio and reduce the uncertainties in the measurement, one should perform the turbidity measurement at a proper incident light wavelength $\lambda$ where the magnitude of $R$ is sufficiently large.

However, for the same sample of suspensions, the coagulation rate constants calculated by eq 4 in turbidity measurement 


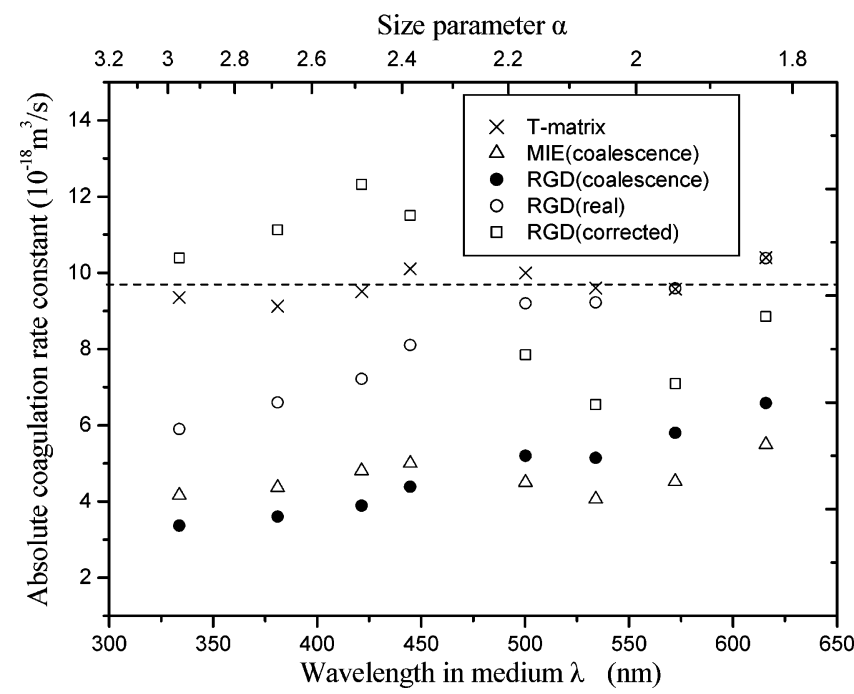

Figure 4. Absolute coagulation rate constants derived from different theoretical treatments for suspensions of particles with $a=170 \mathrm{~nm}$.

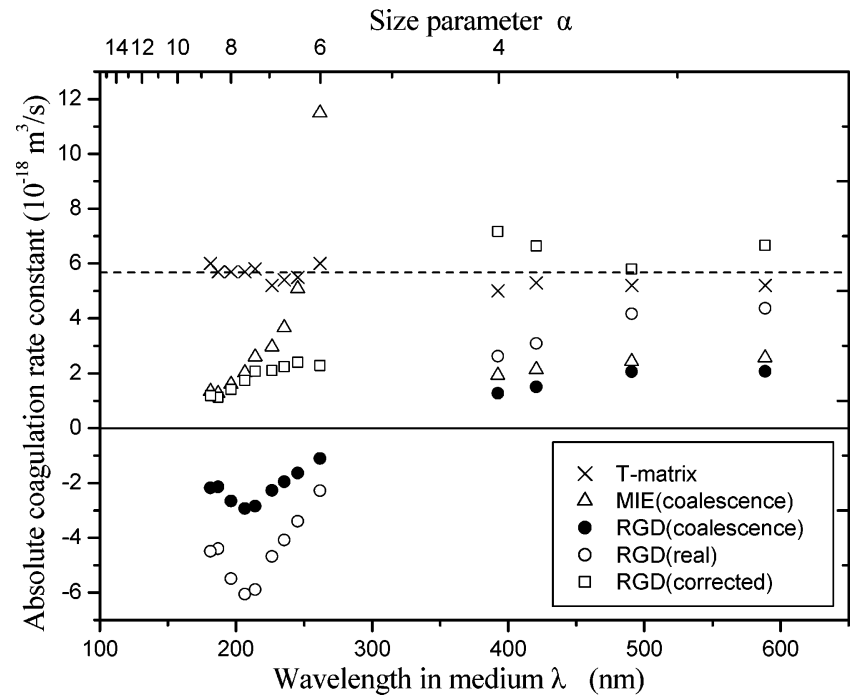

Figure 5. Absolute coagulation rate constants derived from different theoretical treatments for suspensions of particles with $a=250 \mathrm{~nm}$.

performed at different wavelengths have to be unaltered and unique. That means that no matter how dramatically $R$ varies with $\lambda$ the theoretically obtained optical factor $F$ should have the capability of ensuring that eq 4 yields a unique, identical quantity. This point actually suggests a useful criterion for probing the quality of the optical factor $F$ derived from a theoretical treatment.

Instead of evaluating or analyzing them theoretically, we will now use this point as a criterion to experimentally assess the quality of optical factor $F$ derived from the T-matrix method in our study and other theories used before. The results will show that the superiority of the T-matrix method over other existing theories of turbidity measurement is obvious.

All data for the absolute coagulation rate constants determined by eq 4 with the optical factor derived from different theoretical calculations are presented in Figures 4-6. As mentioned above, all points in the same Figure are based on the same original experimental data, namely, the slopes of the linear portion of the $\left(\tau / \tau_{0} \approx t\right)$ curves at different incident light wavelengths, but are derived from different theoretical approaches. In all of these Figures, two different scales are used for the abscissa and are marked by both the wavelength $\lambda$ and the size parameter $\alpha$. Particle sizes of $a=170,250$, and $500 \mathrm{~nm}$ are used in Figures $4-6$, respectively.

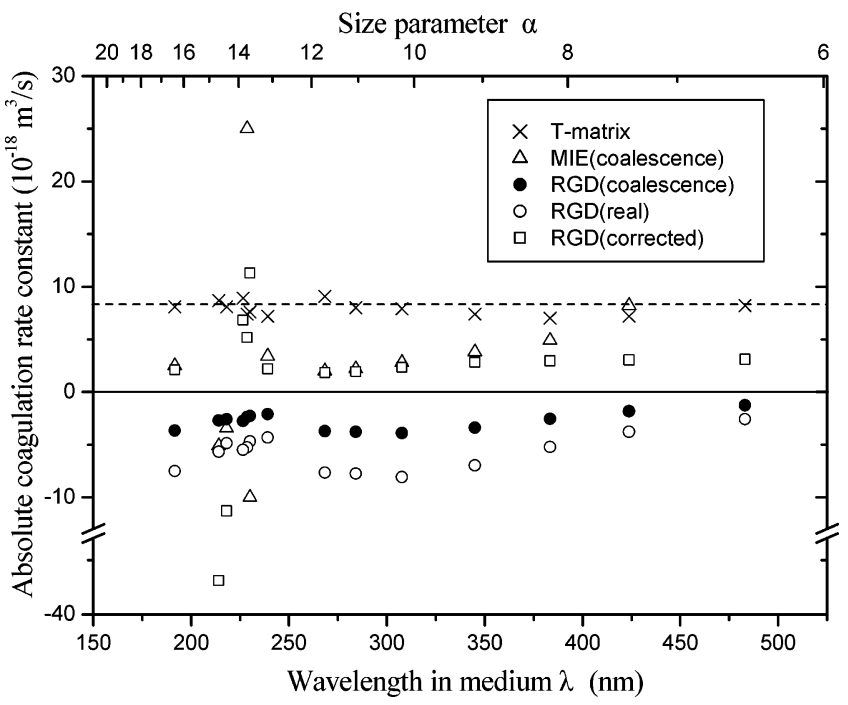

Figure 6. Absolute coagulation rate constants derived from different theoretical treatments for suspensions of particles with $a=500 \mathrm{~nm}$.

These plots show that none of the existing theories can give an unaltered quantity for the whole range of wavelength $\lambda$ or size parameter $\alpha$ used in the experiments. In many cases, the existing theories even yield negative values for the rate constants, which are apparently meaningless. Figures 4 and 5 also show that among all of these existing theories RGD(real) theory performs better when $\alpha$ is small.

However, the validity of the RGD approximation has been tested by several authors,, 19 and the reported error in the determination of the optical factor is about $20 \%$ for $1.5<\alpha<$ 3.0. Because the upper limit for the wavelength obtainable from a commercially available UV-vis spectrophotometer is no larger than $900 \mathrm{~nm}$ (the value in vacuum), the particles that are used have to be small to make $\alpha$ small. For instance, according to the above estimation, if the error in $\alpha=3.0$ is $20 \%$, then to ensure that the errors in the optical factor are $<20 \%$, when using $\lambda=$ $900 \mathrm{~nm}$ the maximum allowable particle radius has to be less than $320 \mathrm{~nm}$ for the aqueous suspension. Note that this is only the error caused by the inaccuracy in the evaluation of the optical factor. When taking into account all of the uncertainties associated with the experiment itself, the total experimental errors may become even unacceptably larger. Thus, to avoid possible errors, the absolute rate constants derived from the turbidity measurement have been limited only to very small particles. ${ }^{8,20}$ Although some researchers ${ }^{21}$ even applied the existing theories for the calculation of absolute coagulation rate constants of the suspensions of particles of $a=1000 \mathrm{~nm}$ by the turbidity measurement and compared the so-obtained data with the Smoluchowski theory, obviously it is difficult to judge the reliability of the conclusion from such a comparison.

From all of the cases shown in Figures 4-6, we can see that the data for the rate constants derived from the T-matrix method basically remain a constant quantity for dispersions of different sizes as well as over the whole range of wavelength $\lambda$ and size parameter $\alpha$ used in the experiments.

Figure 4 (suspensions for particles of $a=170 \mathrm{~nm}$ ) shows that the RGD(real) and T-matrix data are actually overlapping when $\alpha \leq 2$, meaning that for small particles and small $\alpha$ the T-matrix

(19) Kerker, M. The Scattering of Light and Other Electromagnetic Radiation; Academic Press: New York, 1969.

(20) Puertas, A. M.; de las Nieves, F. J. J. Phys.: Condens. Matter 1997, 9, 3313.

(21) Folkersma, R.; van Diemen, A. J. G.; Stein, H. N. J. Colloid Interface Sci. 1998, 206, 482 . 
treatment converges to the RGD(real) one. This fact indirectly proves that the use of the T-matrix solution is correct because it is known that RGD(real) is a good approximation in treating doublets for small $\alpha$ and small particles. In addition, for these particle sizes, all of the data of the rate constants from the four previous theories are basically within the same order of magnitude when $\alpha<3$, although they showing significant deviations from a constant value.

Figure 5 (suspensions for particles of $a=250 \mathrm{~nm}$ ) shows that among four previous theories RGD(corrected) has the best performance and $\operatorname{RGD}$ (real) is second when $\alpha<4$. There are no data available in the $4<\alpha<6$ zone because of the zerosensitivity zone, as mentioned before. When $\alpha>6$, RGD(coalescence) and RGD(real) yield negative values for the rate constants, which are meaningless.

Figure 6 (suspensions for particles of $a=500 \mathrm{~nm}$ ) shows that all previous theories failed to give reasonable data for the rate constant for such sized suspensions when $\alpha>6$. Therefore, the T-matrix solution is the only survivor with excellent performance for the whole $\alpha$ range.

The absolute coagulation rate constant is particularly important for the development of relevant theories or for deriving information on colloidal interactions. However, because of the difficulties with the previous theories in deriving the absolute rates from the turbidity measurement, one usually uses the relative rates of coagulation instead. Indeed, when considering stability ratio $W$, in many cases relative rates of coagulation seem sufficient. Stability ratio $W$ is commonly estimated by the ratio of the diffusion-controlled rapid coagulation rate $\left(k_{\text {rapid }}\right)$ to the coagulation rate $(k)$ of the system under consideration: ${ }^{6,22}$

$$
W=\frac{k_{\text {rapid }}}{k}
$$

However, to obtain $W$, we need a measurement of $k$ for the sample itself, and we also must prepare an additional sample to acquire $k_{\text {rapid }}$. For an unknown suspension, this is not always easy to do. For instance, the methods using eq 8 cannot, in general, be applied to the sterically stabilized systems. ${ }^{6}$ However, the absolute rates do not need two measurements; therefore, they are more desirable if they can be derived accurately from the turbidity measurement.

Thus, the improved turbidity measurement by using the T-matrix method makes it possible to study the coagulation rate and stability with more ease, a broader range of particle size, and improve accuracy.

\section{Conclusions}

We describe a series of experiments to explore the physical features involved in turbidity measurement during the coagulation process. To evaluate the quality of various theoretical treatments dealing with the turbidity measurement to determining the rate constants, we have adopted the criterion based on the fact that optical factor $F$ should ensure that eq 4 yields an identical quantity, which is mentioned above. We believe that this criterion or condition is a necessary requirement for a good theory, even though it may not be sufficient. However, because we know that using the T-matrix approach can treat the light scattering of a real double exactly without a particle size limitation or any approximation, its success in our experimental verification is naturally expected.

However, our experiment also confirms the conclusion reached by other researchers that the turbidity measurement of the rate constant is applicable only for small particles. This study, however, more thoroughly explores the shortcomings associated with the previous theoretical treatments, including the following aspects:

(1) The turbidity measurement of the absolute coagulation rate constant is limited to small particles and small $\alpha$.

(2) There are some inherent problems in the turbidity measurement for small-particle suspension. For small particles, a high number concentration of particles is usually required in order to enhance the signal-to-noise ratio in the measurement. This is because scattering intensity decreases toward smaller particles as the sixth power of the radius and change in turbidity becomes difficult to detect accurately for an almost transparent medium. However, a high number concentration of particles will make the coagulation happen too quickly (Figure 2). Because of the short coagulation time, the linear portion of the curve of $\tau / \tau_{0}-t$ becomes very short before it curves. In this case, the measurement usually needs to be done in several seconds. ${ }^{6,23}$ This causes large errors associated with the data fluctuations due to uneven mixing of the dispersions with salt and the insufficient data collection time. As a step toward improving this situation, Puertas et al. ${ }^{20}$ used the RGD approximation to calculate the total light scattering cross section of the aggregate, taking into account the contributions from pairs of particles with zero, one, and two particles between them, and developed a technique for obtaining the coagulation rate constant from the turbidity measurement by fitting the curve of turbidity change at longer times. However, when large particles with a low number concentration are used, the linear portion of the curve of $\tau / \tau_{0}-$ $t$ can be quite long, as shown for particles of $a=250 \mathrm{~nm}$ in Figure 1, so that the measurement of $R$ may be more accurate.

(3) Because $R$ varies with $\lambda$, to reduce experimental errors one should choose a $\lambda$ at which $R$ is large. If the methodology is only for small particles and small $\alpha$, then one will greatly restrict the choice of $\lambda$.

Here we can see that the previous theoretical treatments are not only valid for the turbidity measurement of the rate constant for a dispersion containing small particles but also have restricted accuracy due to less flexibility in selecting experimental parameters such as $\lambda$ and the coagulation time. Apparently, for more accurate measurements of the rate constant, larger particles are preferable. The shortcomings of the existing theories have greatly limited the applications of the turbidity measurement. Therefore, in many cases, this method is used only to give the rates relative to the rapid coagulation to obtain stability ratios. In contrast to previous theories, the T-matrix approach can provide an exact solution to the calculations of the optical factor required for the turbidity measurement for the absolute rate constant. Therefore, this makes the turbidity measurement applicable to a much broader range of particle size with substantially improved accuracy.

\section{Appendix: T-Matrix Method of Calculating the Extinction Cross Section}

In the T-matrix method, both incident and scattered electric fields are expanded in a series of vector spherical wave functions as follows ${ }^{10}$

$\mathbf{E}^{\mathrm{inc}}(\mathbf{r})=\sum_{n=1}^{\infty} \sum_{m=-n}^{n}\left[a_{m n} \operatorname{Rg} \mathbf{M}_{m n}(k \mathbf{r})+b_{m n} \operatorname{Rg} \mathbf{N}_{m n}(k \mathbf{r})\right]$

(22) Erik, K.Dispersions: Characterization, Testing, and Measurement; Marcel Dekker: New York, 1999.

(23) Sonntag, H.; Strenge, K. Coagulation Kinetics and Structure Formation VEB Deutscher Verlag der Wissenshaften: Berlin, 1987. 


$$
\mathbf{E}^{\mathrm{sca}}(\mathbf{r})=\sum_{n=1}^{\infty} \sum_{m=-n}^{n}\left[p_{m n} \mathbf{M}_{m n}(k \mathbf{r})+q_{m n} \mathbf{N}_{m n}(k \mathbf{r})\right]
$$

where $k=2 \pi / \lambda$ and $\lambda$ is the wavelength. Harmonics $\operatorname{Rg} \mathbf{M}_{m n}$ and $\operatorname{Rg} \mathbf{N}_{m n}$ have a Bessel function radial dependence and are regular (finite) at the origin, whereas functions $\mathbf{M}_{m n}$ and $\mathbf{N}_{m n}$ have a Hankel function radial dependence and vanish at infinity. Because of the linearity of Maxwell's equations, scattered field coefficients $\mathbf{p}=\left[p_{m n}, q_{m n}\right]$ are related to incident field coefficients $\mathbf{a}=\left[a_{m n}\right.$, $b_{m n}$ ] by means of the so-called transition matrix (or T matrix):

$$
\begin{aligned}
& p_{m n}=\sum_{n^{\prime}=1}^{\infty} \sum_{m^{\prime}=-n^{\prime}}^{n^{\prime}}\left[T_{m n m^{\prime} n^{\prime}}^{11} a_{m^{\prime} n^{\prime}}+T_{m n m^{\prime} n^{\prime}}^{12} b_{m n^{\prime} n^{\prime}}\right] \\
& q_{m n}=\sum_{n^{\prime}=1}^{\infty} \sum_{m^{\prime}=-n^{\prime}}^{n^{\prime}}\left[T_{m n m^{\prime} n^{\prime}}^{21} a_{m^{\prime} n^{\prime}}+T_{m n m^{\prime} n^{\prime}}^{22} b_{m n^{\prime} n^{\prime}}\right]
\end{aligned}
$$

In compact matrix notation, eqs $\mathrm{A} 3$ and $\mathrm{A} 4$ can be rewritten as

$$
\left[\begin{array}{l}
p \\
q
\end{array}\right]=T\left[\begin{array}{l}
a \\
b
\end{array}\right]=\left[\begin{array}{ll}
T^{11} & T^{12} \\
T^{21} & T^{22}
\end{array}\right]\left[\begin{array}{l}
a \\
b
\end{array}\right]
$$

which means that the column vector of the expansion coefficients of the scattered field is obtained by multiplying the $\mathrm{T}$ matrix by the column vector of the expansion coefficients of the incident field.

Consider now the computation of the $\mathrm{T}$ matrix for a cluster consisting of $N$ spheres. The total scattered electric field can be written as the sum of the fields scattered by all spheres:

$$
\mathbf{E}^{\mathrm{sca}}(\mathbf{r})=\sum_{j=1}^{N} \mathbf{E}_{j}^{\mathrm{sca}}(\mathbf{r})
$$

Because of the electromagnetic interactions between the components, the total electric field exciting each particle can be written as the sum of the external incident field $\mathbf{E}_{0}^{\text {inc }}(\mathbf{r})$ and the partial fields scattered by all other particles:

$$
\mathbf{E}_{j}^{\mathrm{inc}}(\mathbf{r})=\mathbf{E}_{0}^{\mathrm{inc}}(\mathbf{r})+\sum_{\substack{l \neq j \\ l=1}}^{N} \mathbf{E}_{l}^{\mathrm{inc}}(\mathbf{r}) \quad j=1,2, \ldots, N
$$

The field scattered by the $l$ th particle can also be expanded in outgoing vector spherical wave functions entering at the origin of the $l$ th local coordinate system:

$$
\mathbf{E}_{l}^{\mathrm{sca}}(\mathrm{r})=\sum_{\nu=1}^{\infty} \sum_{\mu=-v}^{v}\left[p_{\mu \nu}^{l} \mathbf{M}_{\mu} v\left(k \mathbf{r}_{l}\right)+q_{\mu \nu}^{l} \mathbf{N}_{\mu} v\left(k \mathbf{r}_{l}\right)\right]
$$

As shown in ref 10, the vector spherical wave functions appearing here can be expanded in regular vector spherical wave functions centered at the origin of the $j$ th reference frame

$$
\begin{gathered}
\mathbf{M}_{\mu \nu}\left(k \mathbf{r}_{l}\right)=\sum_{n=1}^{\infty} \sum_{m=-n}^{n}\left[A_{m n \mu \nu}\left(k \mathbf{r}_{l j}\right) \operatorname{Rg} \mathbf{M}_{m n}\left(k \mathbf{r}_{j}\right)+\right. \\
\left.B_{m n \mu v}\left(k \mathbf{r}_{l j}\right) \operatorname{Rg} \mathbf{N}_{m n}\left(k \mathbf{r}_{j}\right)\right] \\
\mathbf{N}_{\mu \nu}\left(k \mathbf{r}_{l}\right)=\sum_{n=1}^{\infty} \sum_{m=-n}^{n}\left[B_{m n \mu \nu}\left(k \mathbf{r}_{l j}\right) \operatorname{Rg} \mathbf{M}_{m n}\left(k \mathbf{r}_{j}\right)+\right. \\
\left.A_{m n \mu v}\left(k \mathbf{r}_{l j}\right) \operatorname{Rg} \mathbf{N}_{m n}\left(k \mathbf{r}_{j}\right)\right]
\end{gathered}
$$

where vector $\mathbf{r}_{l j}=\mathbf{r}_{l}-\mathbf{r}_{j}$. Explicit expressions for translation coefficients $A_{\text {mnuv }}\left(k \mathbf{r}_{l j}\right)$ and $B_{\text {mnuv }}\left(k r_{l j}\right)$ can be found in ref 10 . Then we can finally derive in matrix notation

$$
\begin{array}{r}
{\left[\begin{array}{l}
\mathbf{p}^{j} \\
\mathbf{q}^{j}
\end{array}\right]=\mathbf{T}^{j}\left(\left[\begin{array}{l}
\mathbf{a}^{j 0} \\
\mathbf{b}^{j 0}
\end{array}\right]+\sum_{l \neq j}\left[\begin{array}{ll}
\mathbf{A}\left(k \mathbf{r}_{l j}\right) & \mathbf{B}\left(k \mathbf{r}_{l j}\right) \\
\mathbf{B}\left(k \mathbf{r}_{l j}\right) & \mathbf{A}\left(k \mathbf{r}_{l j}\right)
\end{array}\right]\left[\begin{array}{l}
\mathbf{p}^{l} \\
\mathbf{q}^{l}
\end{array}\right]\right)=} \\
\mathbf{T}^{j}\left(\mathbf{a}^{j 0}+\sum_{l \neq j} \mathbf{A}^{j l} \mathbf{p}^{l}\right)
\end{array}
$$

where $\mathbf{T}^{j}$ represents the $\mathrm{T}$ matrix for particle $j$, when isolated. The inversion of eq A11 gives sphere-centered transition matrices that transform the expansion coefficients of the incident field into expansion coefficients of the individual scattered fields: ${ }^{13}$

$$
\left[\begin{array}{l}
\mathbf{p}^{j} \\
\mathbf{q}^{j}
\end{array}\right]=\sum_{l=1}^{N} \mathbf{T}^{j l}\left[\begin{array}{l}
\mathbf{a}^{l 0} \\
\mathbf{b}^{l 0}
\end{array}\right] \quad j=1,2, \ldots, N
$$

Finally, the scattered field expansions from the individual spheres will be transformed into a single expansion based on a single origin of the particle system. The incident and scattered coefficients for the system will be derived as

$$
\begin{array}{r}
{\left[\begin{array}{l}
\mathbf{p} \\
\mathbf{q}
\end{array}\right]=\sum_{j} \mathbf{B}^{j}\left[\begin{array}{l}
\mathbf{p}^{j} \\
\mathbf{q}^{j}
\end{array}\right]=\sum_{j, i} \mathbf{B}^{j} \mathbf{T}^{j i}\left[\begin{array}{l}
\mathbf{a}^{i 0} \\
\mathbf{b}^{i 0}
\end{array}\right]=\sum_{j, i} \mathbf{B}^{j} \mathbf{T}^{j i} \mathbf{B}^{i}\left[\begin{array}{l}
\mathbf{a} \\
\mathbf{b}
\end{array}\right]=\mathbf{T}\left[\begin{array}{l}
\mathbf{a} \\
\mathbf{b}
\end{array}\right]=} \\
{\left[\begin{array}{ll}
\mathbf{T}^{\mathbf{1 1}} & \mathbf{T}^{\mathbf{1 2}} \\
\mathbf{T}^{\mathbf{2 1}} & \mathbf{T}^{\mathbf{2 2}}
\end{array}\right]\left[\begin{array}{l}
\mathbf{a} \\
\mathbf{b}
\end{array}\right](\mathrm{A} 13)}
\end{array}
$$

where the $\mathbf{B}$ matrices are similar to the A matrices of eq A11. Now, matrix T so defined is the one needed for the calculations of light-scattering properties of the aggregated particle system. As in our article, what we need is to calculate the extinction cross section of two aggregated particles $C_{2}$ so that $N=2$. The explicit expressions and calculations for the $\mathrm{T}$ matrix can be found in ref 10 .

After getting the T matrix of two aggregated particles, we can get the extinction cross section $C_{2}$ by the equation given in ref 10:

$$
\left\langle C_{\mathrm{ext}}\right\rangle=-\frac{2 \pi}{k^{2}} \operatorname{Re} \sum_{n=1}^{\infty} \sum_{m=-n}^{n}\left[T_{m n m n}^{11}+T_{m n m n}^{22}\right]
$$

In the calculation, two parameters are required: size parameter $\alpha=2 \pi a / \lambda$ and relative refractive index $m=n_{2} / n_{1}$, where $n_{2}$ and $n_{1}$ are the refractive indices of the particle and the medium, respectively. For different wavelengths, the refractive indices, in general, are different. In this article, we use the fitted experimental equations ${ }^{24-26}$ to get the refractive indices of water and polystyrene for different wavelengths.

Acknowledgment. This work is supported by grants 10432060 and 10332050 from the National Natural Science Foundation of China and the Knowledge Innovation Program of the Chinese Academy of Sciences.

LA0602160

(24) Quan, X.; Fry, E. S. Appl. Opt. 1995, 34, 3477

(25) Ma, X.; Lu, J. Q.; Brock, R. S.; Jacobs, K. M.; Yang, P.; Hu, X. H. Phys. Med. Biol. 2003, 48, 4165.

(26) Nikolov, I. D.; Ivanov, C. D. Appl. Opt. 2000, 39, 2067. 